\title{
Research on the morphology of polished surface based on the new polishing method
}

\author{
X.C. $\mathrm{Xu}^{\mathrm{a}}{ }^{*}$ and S.W. Zhang \\ College of mechanical power, Harbin university of science and technology, \\ Harbin, Heilongjiang, China \\ a*1120549159@qq.com
}

\begin{abstract}
Keywords: Polishing. New method. Structure. Simulation. Morphology
Abstract. Because polishing the large die surfaces is very difficult due to its complex machined process, almost all of large die surfaces are manually polished currently, in this paper, researched on a new polishing method for polishing curved surface, simulated the structure of new polishing device with three polishing disks, and compared with linear reciprocating and cross mode; simulated and analyzed respectively surface morphology of polishing workpiece of flat, cylindrical convex and concave surface for different polishing way, and then researched on the impact law on the polished surface roughness about some factors such as the abrasive grain size, the moving speed of polishing head, at last, carried on the test study with the developed new polishing experimental prototype made by the authors of this paper. The research results show that the simulation results agree with test results and simulation analysis is useful to select suitable process parameters for the new polishing machine to polish actual workpiece.
\end{abstract}

\section{Introduction}

Mould industry has gradually become the basic industry of national economy, with the development of industrial technology; the requirement to the mould becomes more and more high. After the large dies are constructed, they cannot make qualified parts due to the design performance of the die surfaces regarding both the complexity of die surfaces and material flow prediction. The die surfaces have to be modified through a series of tryouts until qualified parts are shaped [1]. In such situations, a lot of die surface become free-form surfaces. As for a mass of free-form die surfaces, Surface polishing process is a major operation during die making and tryouts. Currently, almost all of large die surfaces are manually polished, causing low working efficiency and stability of die surface quality, especially in constructions of automotive draw dies of body panels, above situation causes bottleneck regarding the die quality, cost and delivery timing [2]. So, it is a target how to perfect the polishing technology in dies industry [3,4]. This paper focuses on polishing workpiece morphology and its influence factors of polishing surface with the new polishing device by simulation and test.

\section{Simulation about Structure of New Device}

Design of the Polishing Head. Because of the complexity of the metal plastic forming, the mould manufacturing of automotive covering parts needs repeated test and revisions to complete, mold finishing in mould manufacturing, test, and the final production maintenance phase has a very important position, and mould surface grinding and polishing as mould finishing the last procedure, but also plays an important role, the polishing quality directly affects the mold production cycle and delivery time.

At present, hand polishing is a main polishing pattern for large mold polishing, usually there are two kinds of manual polishing mode, one is a kind of reciprocating polishing mode directly, another is a cross polishing mode. A "three-in-one" polishing head has been developed for the prototype machine. This polishing head includes three individual polishing disks that are installed on the three vertexes of the rigid equilateral triangle on the disc base respectively. The three grinding disks have the same size and structure. Each of them can position its own orientation on die surface. The 
polishing disk must always contact die surface during polishing, and the polishing disk cannot intervene with die surface. The polishing disk works with its edge only when polishing concave area, so their edge should be filleted to avoid scribing damages on the die surfaces. For plane and convex area, the polishing disk works with the face, so the center area of the polishing disk should be modified as a concave shape. The polishing disk should be designed in accordance to the concave features, because it can always polish convex and plant features of die surfaces. The interference between polishing disk and die surface has relation with shape and size of die, the head size and sway angle between normal of polishing disk and rotating axis of polishing head.

Simulation about the Structure of New Device. The motion simulation and analyses in this paper are carried out, with the aid of the operation platform of ADAMS and Pro/e software. The parts assemble are designed with Pro/e software, and the motion simulation about the new polishing device is carried out with Pro/e software too. The works finished in this paper with Pro/e are: According to part structure of the new polishing device, the baseline and orders of all kinds of generating characteristics are determined, and reasonable modeling orders are built.

The new polishing device is assembled by pins and cylinder. The simulation analysis is carried out with ADAMS software in this paper. The modeling process is shown in Fig.1.

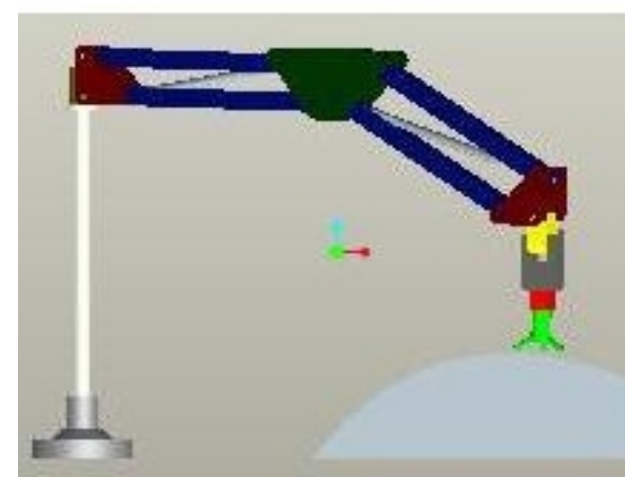

Fig.1 Screenshots of simulation process

\section{Simulation Analysis about the Morphology of Polishing Surface}

Simulation Analysis about Polishing Flat. At present, hand polishing is a main polishing pattern for large mold polishing, usually there are two kinds of manual polishing mode, one is a kind of straight reciprocating polishing way, another is a cross polishing way. Fig. 2 shows the morphology of surface polished straight reciprocating or cross polishing way or three disks with Matlab software. The feed speed is $40 \mathrm{~mm} / \mathrm{s}$. The abrasive is 180 \#, grinder pressure is $0.2 \mathrm{MPa}$, three polishing disks rotation speed is $800 \mathrm{r} / \mathrm{min}$.
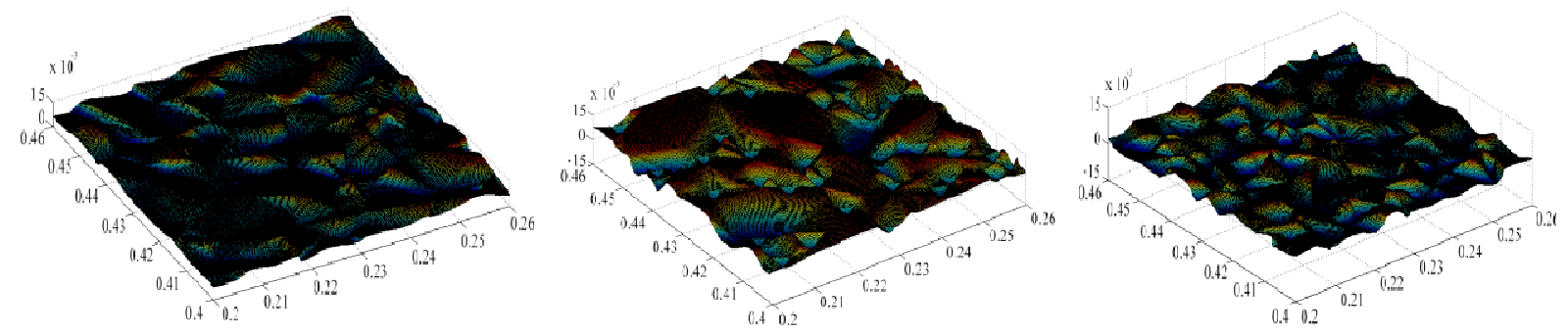

Fig.2 The morphology of flat polished by different polishing way

The polishing morphology of workpiece surface polished by the three polishing discs is the best in the three polishing ways with same process parameters. 
Simulation Analysis about Polishing Cylinder Surface. The surface of the mold can be thought of as consisting of many free curved surfaces, so research about the process of polishing curved surface has more practical significance. In this paper, the study focuses on polishing typical cylinder workpiece. Abrasive granularity is $60 \mathrm{\#}$, polishing pressure is $0.6 \mathrm{MPa}$, the radius of the cylindrical surface is $200 \mathrm{~mm}$. Three kinds of polishing ways: linear reciprocating polishing tools, cross staggered type, screw trajectory motion of three polishing disks. The polishing morphology of convex workpiece is shown as Fig.3. The polishing morphology of concave workpiece is shown as Fig.4 in same process conditions.

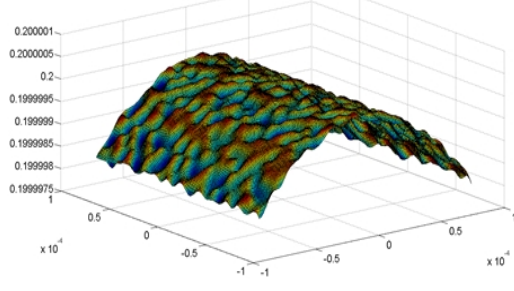

a) linear reciprocating

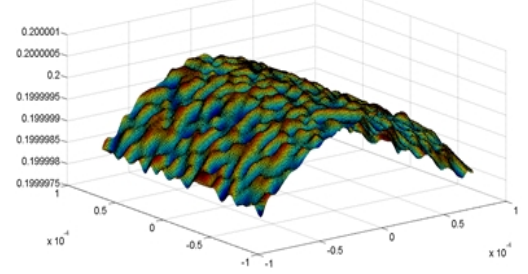

a) linear reciprocating

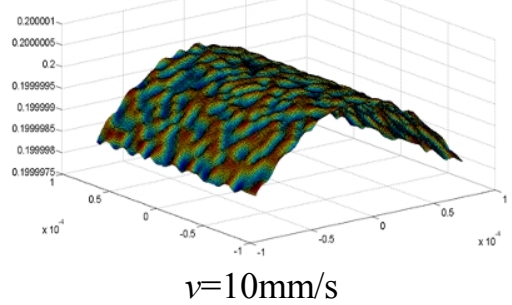

b) cross staggered type

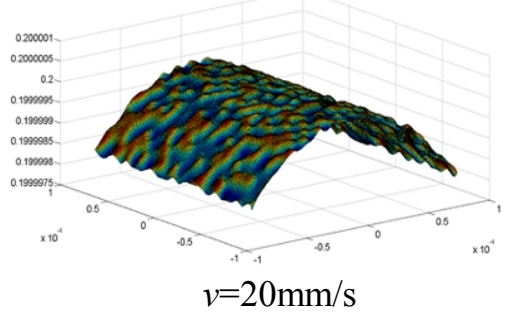

b) cross staggered type

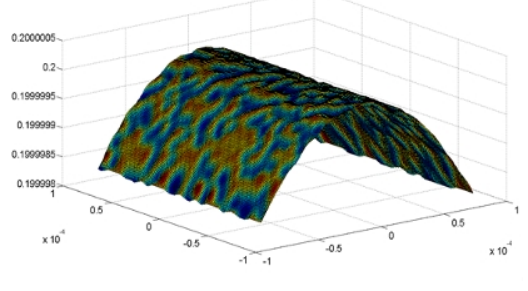

c) three polishing disks

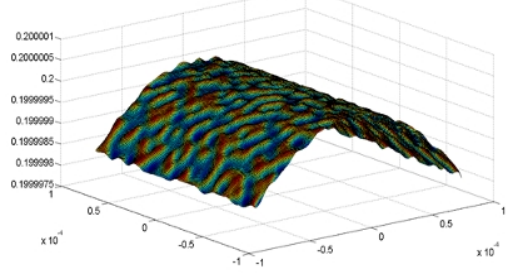

c) three polishing disks

Fig. 3 The morphology diagram of convex workpiece machined surface at different speeds

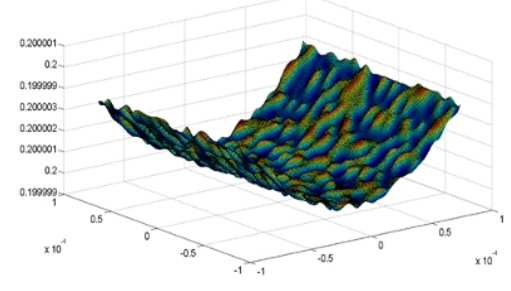

a) linear reciprocating

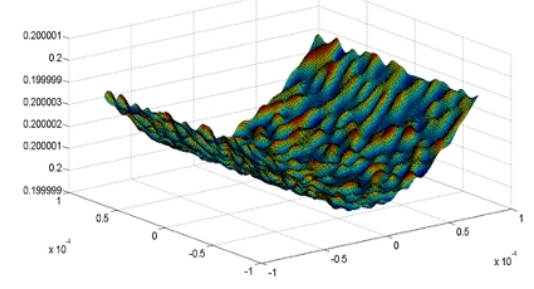

a) linear reciprocating

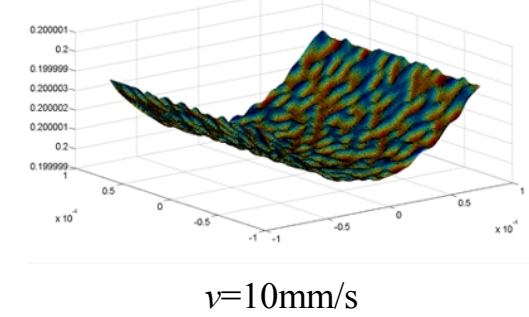

b) cross staggered type

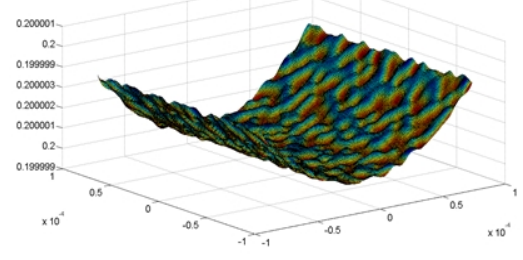

$v=20 \mathrm{~mm} / \mathrm{s}$

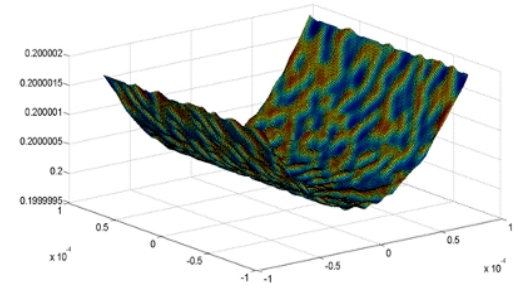

c) three polishing disks

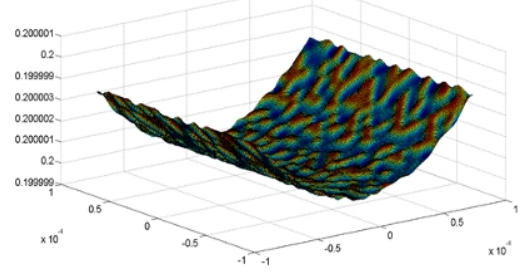

c) three polishing disks

Fig. 4 The morphology diagram of concave workpiece machined surface at different speeds

Shown as Fig. 3 and Fig.4, other conditions remain the same, only change the feeding speed, the shape of the workpiece surface has changed, and the rate of $10 \mathrm{~mm} / \mathrm{s}$ surface after polishing morphology is smoother than that of speed of $20 \mathrm{~mm} / \mathrm{s}$, and the roughness of workpiece surface polished three disks is most small in three kinds of polishing ways. 


\section{Test Analysis}

The above simulation results show that the effect of polishing with three disks is better than the other polishing ways, so this paper only made polishing test with three disks in order to inspect simulation results. Fig.5 shows simulation results agree with test results. This error may be caused by simulation assumptions about grinding grain of uniform distribution.

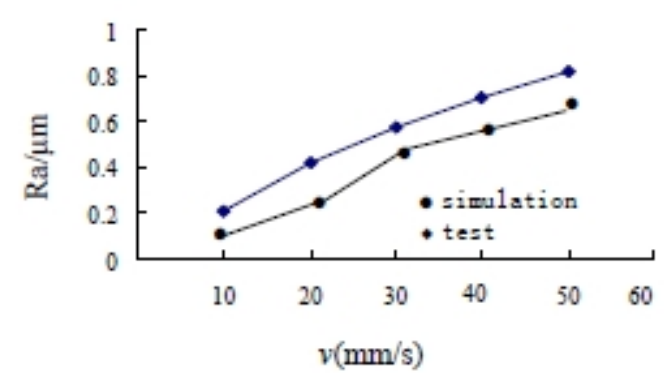

Fig. 5 The impact of speed in polishing convex cylindrical

\section{Summary}

In this paper, a new polishing device and the morphology of polishing surface were simulated analysis. Some affecting laws of some factors about polishing surface roughness and morphology were analyzed such as the grain size of polishing disk, feed speed. Through simulation analysis, when polishing cylinder, the smaller grinding grain of polishing disk is, the smaller the feed rate is, the better the morphology of the workpiece surface is. The simulation results show that the effect of polishing with three disks is better than the other polishing ways.

The simulation results agree with test results that proved by polishing cylindrical convex and cylindrical concave, the research results show that simulation analysis is useful to select suitable process parameters for the new polishing machine to polish actual workpiece.

\section{References}

[1] Altan and T.J. Mater: Processing Technology, No.59 (1996), pp.158-163.

[2] W.M. Chan, L. Yan, W. Xiang: The International Journal of Advanced Manufacturing Technology, Vol.22 (2003), pp.387-390

[3] L.N. Yu, Y.L. Li: Journal of Hunan Industry Polytechnic, Vol.10 (2010), pp.8-11, in China

[4] C.Y: Henan Agricultural, No.3(2013), pp.55-59, in China 